\title{
Numerical and Experimental Characterization of RF Waves Propagation in Ion Sources Magnetoplasmas
}

\author{
G. Torrisi, D. Mascali, G. Sorbello, G. Castro, L. Celona, Member, IEEE, and S. Gammino
}

\begin{abstract}
This paper describes three-dimensional numerical simulations and Radio Frequency (RF) measurements of wave propagation in microwave-heated magnetized plasmas of ion sources. Full-wave solution of Maxwell's equations has been addressed through the Finite Element Method (FEM) commercial software COMSOL. Our numerical model takes into account the strongly inhomogeneous and anisotropic magnetized "cold" plasma medium. The simulations reproduce the main features of the wave-plasma interaction of the Flexible Plasma Trap (FPT) that recently came into operations at INFN-LNS. A two-pins $\mathrm{RF}$ probe has been ad-hoc developed and used as a plasmaimmersed antenna for measuring local wave electric fields in the FPT device. The measurements of plasma electron density and RF electric field, performed for different external magneticfield configuration, allowed a direct comparison with the assumed simulation model.
\end{abstract}

Index Terms-Radio wave propagation, Plasma waves, modeling, $\mathrm{RF}$ heating, RF diagnostic, RF probes, $3 \mathrm{D}$ electromagnetic field simulations

\section{Introduction AND Motivation}

$\mathrm{R}$ ADIOFREQUENCY propagation of electromagnetic waves through plasmas [1], [2], has gained considerable interest in many fields: broadcast communication space exploration [3], plasma sheath in reentry vehicles [4], thermonuclear fusion [5], plasma processing [6], microwave plasma diagnostics and heating in ion sources [7]-[10]. In particular, we focus on the mechanism of RF propagation into the nonhomogeneous magnetized anisotropic lossy plasma of Electron

Cyclotron Resonance Ion Sources (ECRIs) and Microwave Discharge Ion Sources (MDIS) [11]-[13].

The issue of radiofrequency propagation in anisotropic plasmas confined in compact traps such as ion sources is still a hot-topic in the relative scientific community. No models and results from numerical approaches exist in literature providing a clear and reliable description of wave launching, propagation and absorption in the small-size (compared to the wavelength) magneto-plasma. The point of a direct comparison to experimental data, in particular to spatially resolved measurements of the in-plasma electric field, is also of particular importance, since it could represent a step towards the development of numerical tools becoming more and more predictive.

In this paper, the electromagnetic wave propagation in microwave-heated plasma confined in a magnetic field, is

The authors wish to thank the 5th Nat. Comm. of INFN, under the Grant PANDORA for the financial support.

G. Torrisi (e-mail: giuseppe.torrisi@lns.infn.it), D. Mascali, G. Castro, L. Celona, S. Gammino are with the Istituto Nazionale di Fisica Nucleare Laboratori Nazionali del Sud, Via S. Sofia 62, 95123 Catania, Italia

G. Sorbello is with Università degli Studi di Catania, Viale Andrea Doria 6, 95125, Catania, Italia and also with the Istituto Nazionale di Fisica Nucleare - Laboratori Nazionali del Sud, Via S. Sofia 62, 95123 Catania, Italia addressed through numerical simulations and experiments, devoted to understand how the RF wave propagation depends on the electron density profile and external magnetic configuration which can be often controlled in many kind of plasma physics and application experiments.

A better comprehension of electromagnetic wave propagation and RF power to plasma coupling mechanisms in ionsources magnetoplasmas is crucial in order to provide a costeffective upgrade of these machines alternative to the use of higher confinement magnetic fields, higher RF power level and higher pumping wave frequency [14].

Three-dimensional self-consistent numerical simulation of full-wave RF fields in inhomogeneous non-uniformally magnetized plasma is a big challenge [15], [16] and only few methods have been developed to obtain self-consistent solutions [17], [18]. Several full-wave numerical simulation codes have been developed, exploiting ray-tracing technique or finitedifference time-domain (FDTD) method [19]-[22]. Alternative models and numerical strategies have been attempted by other authors by assuming assuming "zero-dimensional" up to 2Dsimulation model in a simplified magnetostatic scenario with an axial symmetry or by considering the plasma medium as an equivalent dielectric load [23], [24]. However, the wave propagation at frequencies in the range $3-40 \mathrm{GHz}$ in ECRIS compact plasma (having magnetic field of the order of few Tesla, electron density in the order of $10^{18} \mathrm{~m}^{-3}$ ) cannot be predicted by the plane wave model nor addressed by "raytracing". These models, in fact, fail in minimum-B configuration scenarios where the scale length of plasma nonuniformity, $L_{n}=\left|n_{e} / \nabla n_{e}\right|$, and the magnetostatic field nonuniformity, $L_{B_{0}}=\left|B_{0} / \nabla B_{0}\right|$, are smaller than the free space, $\lambda_{0}$, and guided, $\lambda_{g}$, wavelengths. The Wentzel, Kramers and Brillouin (WKB) method - often adopted to describe waves in fusion toroidal devices [25]-[27] - is also not applicable because the wavelength is comparable with the plasma gradient and dielectric tensor scale lengths. Only full-wave methods are suitable to capture waves' propagation features, strongly affected by the resonant cavity leading to the formation of electromagnetic standing wave modes.

Hereinafter we present numerical results of $3 \mathrm{D}$ simulations of $\mathrm{RF}$ wave propagation in the magnetized plasma of the "Flexible Plasma Trap" (FPT) [28], an ion source test-bench developed on purpose at INFN-LNS, to testing novel RF systems for plasma heating and diagnostic schemes. We used COMSOL Multiphysics [29] software to model a "cold", anisotropic magnetized plasma, described by full-3D non uniform dielectric tensor, enclosed by the metallic cylindrical cavity where the plasma is generated. A proper mesh generation, exploiting FEM-based COMSOL versatility, allowed us 
to optimally modelize the cavity and microwave waveguide launching structure, with a good computational efficiency and high resolution especially around the resonance regions. The main goal of the paper is to directly compare, for the first time in ECR ion sources, the numerical models results with RF measurements of the wave amplitude inside the FPT plasma chamber, performed through a two-pin RF probe antenna.

Measurements of field structure correlated with plasma density, magnetic profile and electron temperature have been already presented up to now only for radially localized helicon (RLH) waves detection on helicon plasma sources [30]-[32] and for direct detection of Electron Bernstein Waves (EBW) in large volume magnetoplasmas of toroidal fusion plasma reactor [33]-[35]. In the latter case the pumping wavelength $\lambda \ll L_{n}, L_{B_{0}}$. On the other hand, in ECRIS complex environment, the strong interaction of the resonant cavity with the electromagnetic wave propagation affects the polarization state and no a priori assumption on the polarization state can be done. RF measurements in small volume magnetoplasmas are especially needed in order to investigate well beyond the assumed oversimplified damping mechanism of the right-hand polarized wave (RHPW) absorption at the ECR layers.

The article is arranged as follows. Section II is on wave field solutions of the Maxwell's equations taking into account the magnetic field which makes plasma anisotropic, nonuniformity of plasma density, and the metallic plasma chamber. The section III describes the experimental setup. Finally the results of the numerical analysis are presented in Sec. IV and compared with experiments.

\section{RF WAVE-PLASMA INTERACTION MODELING}

In compact ECR plasma machines, the magnetically confined plasma is ignited by microwaves in the $\mathrm{GHz}$ range resonantly interacting with free-electrons in a rarefied gas (down to $10^{-8}$ mbar) via Electron Cyclotron Resonance, occurring when $\omega_{R F}=\frac{\omega_{\mathrm{e}}}{m_{\mathrm{e}}}$, where $m_{e}$ and $e$ are the electron mass and charge, respectively.

A magnetized plasma in the GHz range frequencies can be modeled as a cold magneto-fluid with collisions where the field-plasma interaction is described by the tensorial constitutive relation $\bar{Q} \cdot E$. Tipically $\bar{Q}$ is derived assuming a magnetostatic field $B_{0}$ directed along just one axis. This assumption is valid in most of cases but not in ECRIS where $B_{0}$ is not strictly axis-symmetric. Considering the actual magneto-static structure of an ECRIS, that is not uniform nor axis-symmetric, $\bar{Q}$ depends in a complex way from the magnetostatic field $B_{0}(x, y, z)$ and the local electron density $n_{e}(x, y, z)$. Under the "cold plasma" approximation, (i.e. $\mathrm{v}_{\varphi} \gg \mathrm{V} t$, being $\mathrm{V}_{\varphi}$ the wave's phase speed and $v_{t h}$ the electron thermal speed), the tensor components have been calculated in [13] where it is shown that the tensor $\bar{Q}(x, y, z)$ exhibits a local dependence from the external static magnetic field, $B_{0}(x, y, z)$, and plasma density, $n_{e}(x, y, z)$ :

$$
\overline{\overline{Q_{r}}}(x, y, z) \Rightarrow \overline{\overline{Q_{r}}}\left(B_{0}, n_{e}\right)
$$

Eliminating the magnetic field between Maxwell's equations and using the above constitutive relation (1) for an anisotropic medium, the wave equation reads as:

$$
\nabla \times \nabla \times E-\frac{\omega^{2}}{c^{2}} \overline{\overline{Q_{r}}} \cdot E=0
$$

The above wave equation (2) can be solved as a driven problem by a FEM solver that supports a non homogeneous tensorial constitutive relation; in the present work we used COMSOL and an external MATLAB routine allowing the definition of the full 3D dielectric tensor. For sake of clarity, the simulations

hereby described are based on the "stationary" frequency domain COMSOL solver since general properties of an ECR plasma can be considered stationary on very long timescales.

In COMSOL we were able to model the exact shape of the FPT plasma chamber and the waveguide launching structure.

While the static magnetic field, $B_{0}$, is externally imposed by the current flowing in the magnetic system and can be both accurately predicted and measured (and for this reason can be considered a known input parameter), on the contrary, the plasma density, $n_{e}$, depends from the local electromagnetic field, $\{E, H\}$, that sustain the plasma. In other words the anisotropic wave equation (2) and constitutive relations (1) are in principle a system of coupled equations. The coupled problem can be solved by an open loop approach [13] assuming a reasonable plasma density and or in a self-consistent approach [18].

In this work we overcome the above approaches and we adopt the empirically meeasured magnetic field and the plasma density. Then, for the first time, we compare the numerically predicted electromagnetic field, $\{E, H\}$, with the measured one. The validation has been carried out on a particular machine (the FPT), however it should be noted that the approach remains of very general interest since the constitutive relation are local (1) and valid for any anisotropic cold plasma. Moreover, the FPT allows a wide range of working points that have been explored in the experimental validation carried out (see below). Since in proximity of the resonance surface (individuated by the iso-surface $B_{0}=B_{E C R}=\frac{m e}{e \omega}$ ) the permittivity varies strongly, the discretization of such a narrow region needed a very fine mesh: to achieve this, we adopted an adaptive mesh procedure (that was allowed by a specific feature of the solver). An extremely fine mesh has been obtained, thanks to "functional evaluation" based on the electric field gradient. In the simulations, the experimental setup is modeled by the computational domain shown in Fig. 1. The domain consists of the cylindrical plasma chamber cavity and microwave double ridged injection waveguide operating in the fundamental $\mathrm{TE}_{10}$-like mode to allow a wide bandwidth (from $3.5 \mathrm{GHz}$ to $8.20 \mathrm{GHz}$ ). The inner cavity volume is filled by lossy plasma characterized by dielectric tensor. The cavity walls are modeled via the appropriate "Perfect Electric conductor" boundary condition. Table I reports the Simulation input parameters.

\section{EXPERIMENTAL SCENARIO AND RF PROBE ANTENNA DESCRIPTION}

The Flexible Plasma Trap (FPT) installed at INFN-LNS and described in [28] consists of a copper cylindrical plasma 


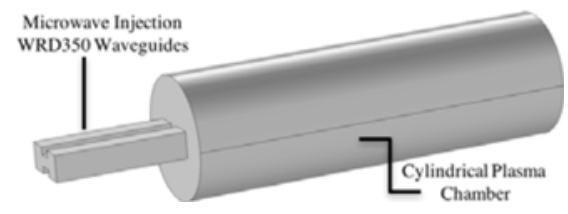

Fig. 1: Simulated Geometry: Cylindrical plasma chamber cavity and microwave rectangular double ridge waveguide injection

TABLE I: Simulation input parameters.

\begin{tabular}{|l|l|l|}
\hline Parameter & Value & Description \\
\hline $\mathrm{L}$ & $250[\mathrm{~mm}]$ & Cavity length \\
$\mathrm{R}$ & $65[\mathrm{~mm}]$ & Cavity radius \\
"RF & $6.827[\mathrm{GHz}]$ & Frequency \\
Weff & $w r c p / 10^{3}[\mathrm{racVs} 1$ & Co llision frequency \\
Prep & $100[\mathrm{~W}]$ & RF Power \\
\hline
\end{tabular}

chamber $250 \mathrm{~mm}$ long and $65 \mathrm{~mm}$ in radius, having numerous flanges for pumping, gas inlet, diagnostic and three RF rectangular input waveguide po rts for test different plas ma heati ng sc hemes. Figure 3 shows a schematic diagram of the FPT machine, the RF power delivery system, the three magnetic coils, and diag nos tic probes (RF probe for electric field detection or Langmuir Probe for electron density measurement). The FPT plasma has been fed by continuous mode microwaves at the operating freq uency of $6.827 \mathrm{GHz}$ generated by a TWT Microwave Amplifier. An isola tor protect s the TWT from any reflected power and a dual directional coupler is used for measuring the forward and reflected powers. Microwaves are then guided via WRD350 double ridge rectangular waveguides and through a Kapton window into the vacuum chamber which maintains the vacuum integrity. Plasma is confined by a static magnetic field obtained by means of three coils, placed coaxially to the plasma chamber and able to generate different magnetic field profiles. As it is poss ible to see in Fig. 3, one of the chamber endplates is dedicated to microwave launching and gas-i nec tion, the other one to plasma diagnostics. The ex periments are performed at wave input power $P ; n=l O O \mathrm{~W}$ at the operating frequency of $6.827 \mathrm{GHz}$ and at the pressure of $1.5 \cdot 10-{ }^{4}$ mbar. We used three different configuration for the external magnetic field leading to three "simple mirror"- like profiles .

A ce ramic-coated RF two-pins probe small antenna, with o ne pin grounded and another connecte $\mathrm{d}$ to the inner wire of a coaxial transmission line, has been developed and used to measure the wave electric field inside FPT plasma chamber. In principle, we could use a si mple se mi rigid coaxial cable with a coaxial pin that protrudes from the dielec tric ins ulatio $n$ (low density dielectric Teflon ${ }^{\circledR}$ PTFE). However, a two-pins probe antenna allows to measure the poten tial difference (or the E-field component) perpendicular to the pins resulting in polarization selectivity as demostrated in [35).

The pins length and distance have been designed to be sensitive to the short wavelengths and small enough to achieve the desired spatial resolution. The probe is constructed out of Suco flex $\odot$ microwave $0-40 \mathrm{GHz}$ cable, having a silver- plated copper inner conductor with diameter of $1 \mathrm{~mm}$ and an outer diamete $\mathrm{r} 4 \mathrm{~mm}$. The probe exposes two linear tips having length of $3.5 \mathrm{~mm}$ and a distance of $2 \mathrm{~mm}$, and it is enclosed in a high-purity alumina protection tube allocated parallel to the longit udinal axis plasma chamber. The probe is connected to vacuum $\mathrm{RF}$ feedthro ugh $\mathrm{K}$-connector that was mounted on a standard CF -40 flange. A bellow allows movements via computer controlled step motor which provides a $0.1 \mathrm{~mm}$ precision. In the current set up (see Fig. 3), the probe can penetrate into the plasma chamber along a line pa rallel to the chamber axis. The RF probe pins a re pro tec ted from direct contact with the plasma by a coating of MACOR. The output from the RF probe is fed into Power Probe. The new electric field probe has many desirabl e features such as high se nsit ivity, good spatial resolution, polarizatio $\mathrm{n}$ se ns itivity, and proven reliability in a high temperature enviro nment. Together with the already existing diag nostics for X-ray, optical e missio n, electron density, it gives the possibility to characterize plas ma also in the RF electromagnetic spectrum.

\section{NUMERICAL AND E XP ERIMENTAL R ESULTS}

$\mathrm{Fu}$ ll-wave simulations were carried out through the COM SOL Multiph ysics FEM solver, considering the geometry shown in Fig. I and the above described "cold plas ma" model. Our three-dimensional 3D RF field solver uses the experimentally measured plas ma density, $n e(x, y, z)$, and magnetosta tic, $B_{0}(x, y, z)$, profiles as input functions. Figure 2 disp lays three different cases of electron density ne measured through a Langmuir probe [14) and obtained for three different values of

the ratio $\frac{8}{8} m^{\prime \prime \prime}$ between the minimum magnetic field, Bmin, and the value of magnetic field on the resonance surface, $B E C R$ •

In particular, concerning the plasma density measurements, they have been obtained alo ng a line of view that is a lmost parallel to the plasma chamber axis. Regarding the radial pro files, at the moment we do not have direct access for measuring the de nsity along a given line of sight. Anyway, in simple mirror devices, co nsiderin g basic assumptions about plasma diffusion across the magnetic field, a cos (r ) trend ca $n$ be reaso nably argued and assumed for radial density trend as suggested in (36), and it is in ge neral agreement with the optical ins pec tion of the plasma and with X-ray imaging (37].

The mag netic field map has been experimentally obtained meas uring the magnetic inductio $\mathrm{n}$ by means of a commercial "Lake Shore Cryotronics, Inc." Hall probe. The field has been measured in axis andoff-axis position, both for axial and radial

components, then the 3D map can be easily reconstructed

taking profit from the symetry of the system. In particular, we study three working points differing for the magnetic field configurations:

I ) Simp le mirror configuration with a $\mathrm{Z}$-distance between ECR layers $W E C R$ (where $B O=B E C n$ ) of $70 \mathrm{~mm}$ and a ratio of the minimum magnetic field $B m i 7 i$ ove $\mathrm{r}$ the $B E C R, f ;, ;=0.65$. 


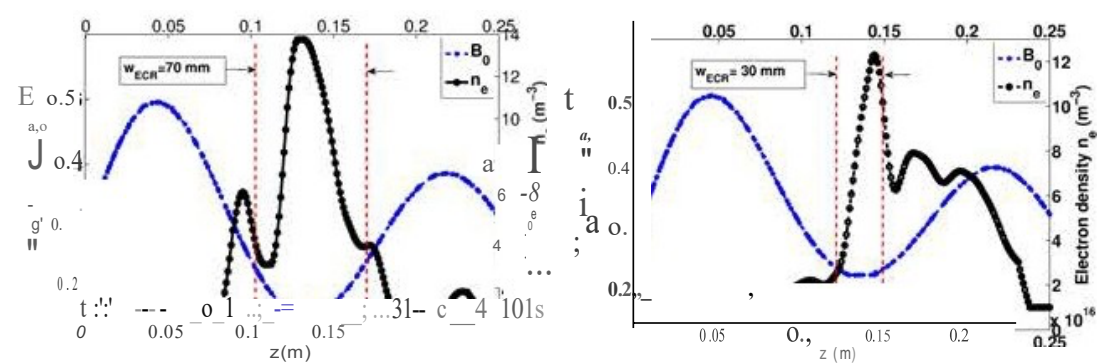

(a) Case I (w ee $n=70 \mathrm{~mm}$ ), Input param- (b) Case 2 (wsc $n=30 \mathrm{~mm}$ ), Input parameters: black point marker curve represents eters: black point marker curve represents

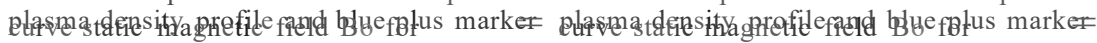
0.65 . $n$

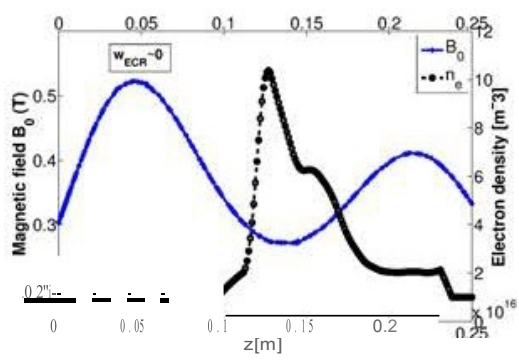

(c) Case 3 (ween $0 \mathrm{~mm}$ ), input pa-

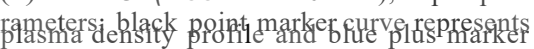
curve static magnetic field Bo for $\frac{8}{0}=-{ }_{-}=1$.

Fig. 2: Measured electron density and magnetic pro file along the the z-axis; this data is used as input parameter for the simulations, the positions of the ECR layers are indicated with vertical red dashed lines.

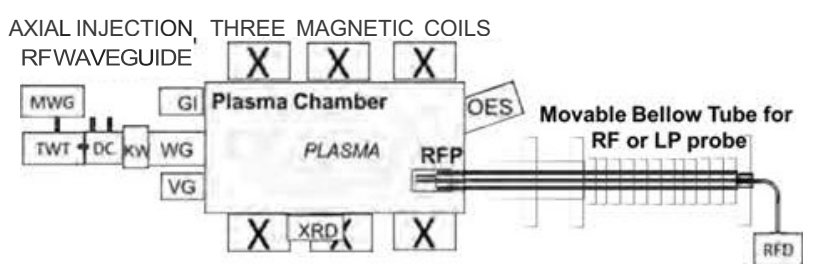

Fig. 3: Schematic of the FPT experimental setup at the INFNLNS. MWG: 1-20 GHz Microwave Generator. TWT: 500 Watt 4-7 GHz Traveling Wave Tube Amplifier, DC: directional coupler, KW: Kapton window, WG: waveguide sec tion, VG: Vacuum Gauge, GI: Gas Injection, RFP: Radio Frequency P robe, LP: Langmuir probe, XRD: X-Ray detec tor, OES: Optical Emission Spectroscopy, RFD: Radio Frequency detector DC-44 GHz.

2) Simple Mirror configuration with $W E C R=30 \mathrm{~mm}$, Wpwer,
Simple Mirror

3) Simple Mirror configuration without ECR layers and

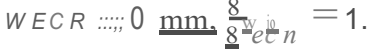

For eac h working point, the "cold" pem1ittivity tenso $r$ for 3D full-wave simulation s was derived and used.

In order to explore the different propagation characteristics in terms of diss ipated power (RF power absorbed by plasma) we can see the simulation results in figures $4 \mathrm{~b}$ and $4 \mathrm{c}$. The dissipated power - plotted on $x z$ plane and along the line of sig ht of the RF probe - allows a comparison of the power absorption for the three magnetic field configurations listed above. Both ID and 2D plots show that the power is mostly deposited along layers, which become closer and closer when increasing the $\frac{811}{8 E} \sum_{R}$ ratio. $2 \mathrm{D}$ plots point out the structure of the absorpt ion laye rs which is strong ly modified by the ratio as well. rn particular, the model is able to capture the physics involved, since absorption clearly occurs where ECR layers are placed at each ratio, as it is clearly shown in Fig. 4a.

The direct com parison betwee $\mathrm{n}$ simulations and measurements demonstrate that the COMSOL simulated RF field pro- file clearly resembles the experime ntally measured ones (see Fig. 5) for the three magnetic fields configurations showed in Fig. 2. Figures 5 show good agreement between the measured and full-wave FEM simulated field intensity $\left.\mathbb{E}\right|^{2}$. What it is relevant is that for the configuratio ns at larger WEC R, the elec tromagnetic field see ms to self-co nfioe in the plasma volume eoclosed by the resonance layers. Th.is "plasma-cavity" effect was already predicted in [13], [38] occ urring under specific co nditions of plasma density and sizes. It is now confirmed also by the direct RF measurements. The amplitude peaks near the ECR layers confirm that the presence and position of the ECR layers can be predicted by the FEM calculatio ns. It is however remarkable that - regardless from the absolute height of the single peaks - the simulations very well rep roduce the peaks-valleys sequence (which is a direct consequence of wavelengths scale in the plas ma filled cavity). It appears very clear that the agreement betwee $\mathrm{n}$ experimental aod numerical data is better when the plasma size is the largest one, while fo $r$ the $W E C n \quad \ldots, \ldots, 0$ case the profiles tend to deviate each other. In pa rticul,ar measurements show that the electromagnetic e nergy $\mathrm{s}$ tarts to "escape" from the inner resonance volume as $w$ e $c R$ becomes smaller and smaller. $\mathrm{T}$ his e ffect is only partially reproduced by the si mulations. It is worth mentio ning, anyway, that the configurntion $w s c n$-t $\mathrm{O}$ is very rarely adopted in plasma traps and was he re mentioned as a critical co ndition for checking the model reliability under different magnetic field profiles. $\mathrm{T}$ he agreement is higher as highe $\mathrm{r}$ it is the plas ma density within plasma chamber. This is probably caused by the perturbing presence of the radio frequency probe.

Up to now, simulations results are without probe; we know the perturbation introduced from the probe on the electromagnetic field strongly depends on the probe position. This in principle implies that we should perform one simulation for each probe position, which will be done in future works. For the moment we performed ooe additional simulation by considering also the presence of the probe for the case 2, ME $c n=30 \mathrm{~mm}, \underline{\beta}_{\text {niiu }}=0.92$ ) at a fixed position $z=0.2 \mathrm{~m}$. in Fig. 6, we compared the simulated electric field with and without probe. Due to the presence of the insulating dielectric 


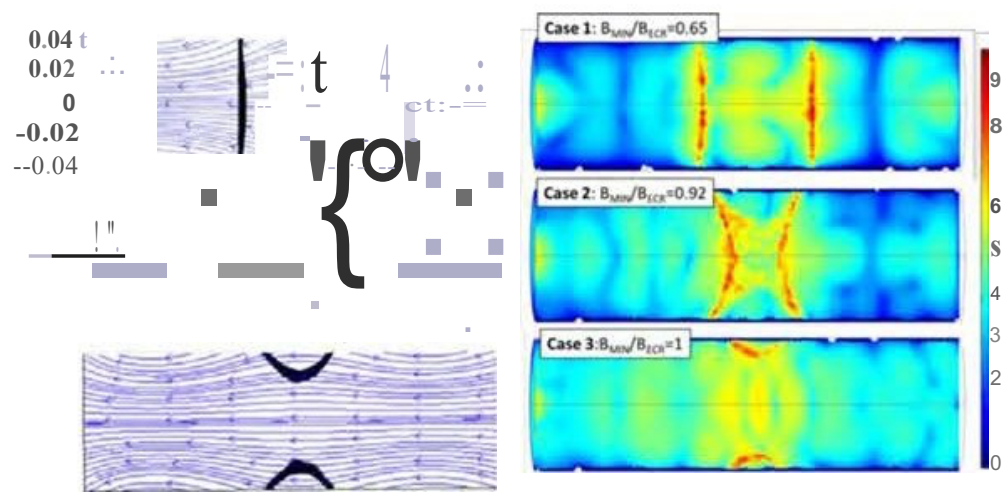

(a) Magnetic field lines and resonance (b) Power Loss Density IW/m³ ${ }^{3}$ (log scale) layers (black surfaces) on xz plane

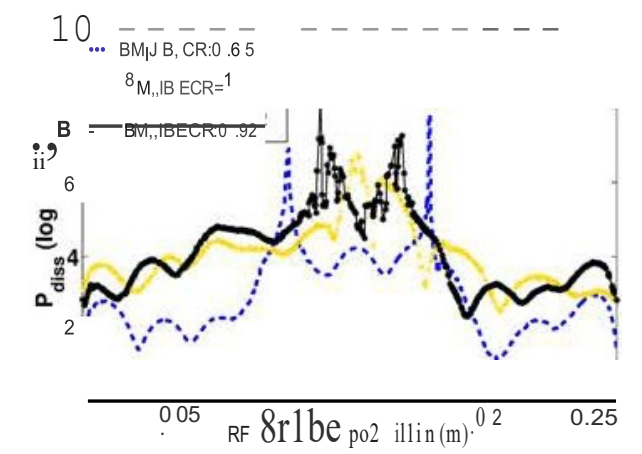

(c) Power Loss Density $\left[\mathrm{W} / \mathrm{m}^{3} \mathrm{~J}\right.$ (log scale) along the line of sight of the RF probe

Fig. 4: Simulated Electromagnetic Power Loss Density $\left[\mathrm{W} / \mathrm{m}^{3}\right]$ (log scale) in plasma chamber.

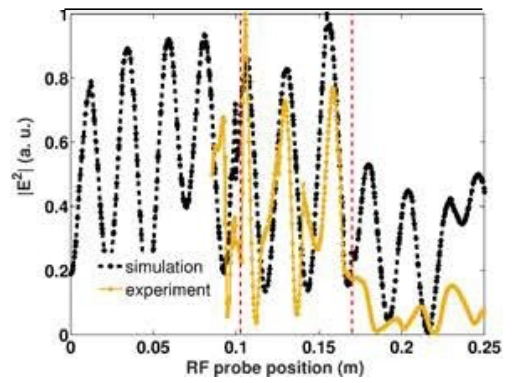

(a) Case I: ween $=70 \mathrm{~mm}$. $=0.65$. This is the case with the largesf i 11 sma volume enclo sed by the ECR layers. There is an excellent agreement between s imulated and measured RF field distribution.

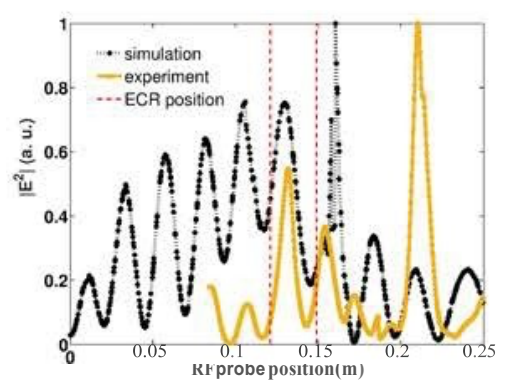

(b) Case 2: wse $n=30 \mathrm{~mm},-f l=-=0.92$.

c) Case 3: ween --, 0, ffUW-- = 1. Thi ithesents a critical configuraf'i6, very rarely is becoming smaller. The agreement between tuned in ECR machines. I.n this case there is simu lated and measured RF field distribution still a general agree ment even if locally the is still evident.

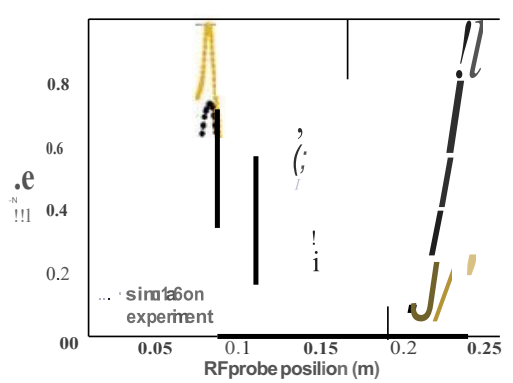

two plots deviate somewhere.

Fig. 5: Simulated and measured RF electromagnetic field along the probe position.

of the coaxial probe, an attenuation of the field can be noticed as it is evident also in the experimenta 1 results of Fig. 5 (b). For lower electron densities (case 3), the probe influence on the cavity mode dominates. When density increases, the modal behavior becomes more and more sensitive to the plasma presence within the chamber, whose influence is predicted by the FEM simulations. Therefore, the agreement between FEM predictions and experimental measuremems increases (case I).

\section{C ONCLUSION}

The paper has reported about the direct comparison of numerical results (from a full-wave approach) with the inner plasma measurements of the elect ric field amplitude performed 


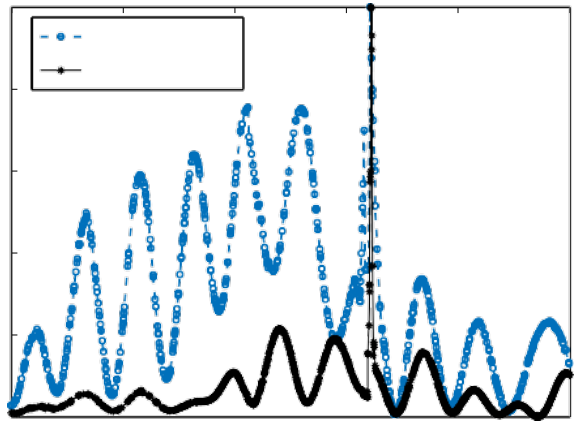

Fig. 6: Simulated Elecric field for Case 2 with and without probe.

by means of a two-pins RF probe. Both simulations and measurements have been done considering a compact plasma trap (resembling very closely the features of any ECR ion source) in a simple mirror configuration. The resulting code that propagate the electromagnetic fields into a cold anisotropic plasma and its validation against experimental data is very accurate and of general effectiveness: we showed that the cold plasma model (1) captures the essential features of the wave propagation in plasma when both the static magnetic field and the plasma density are accurately estimated and/or tuned on experimental data. The excellent agreement between model predictions and experimental data are very promising for the design of future launchers or "exotic" shapes of the plasma chambers in compact machines, such as ECR Ion Sources and other similar devices. Our approach - integrating a FEM commercial solver and a self-made MATLAB developed routine for local tensor computation - starts to shed some light on the electromagnetic problem of propagation and absorption in magnetized plasma. Hence the results, even if benchmarked on a particular machine, have actually a general validity, at least for every RF-heated magneto-plasma compact device or wave propagation in magnetized plasma, in cold approximation. Further steps forward are going to be done as concerning the improvement of the model, including the "hot" plasma tensor: this will perspectively allow to master additional mechanisms such as modal conversion at the hybrid resonances.

\section{REFERENCES}

[1] T. Stix, Waves in Plasmas. American Inst. of Physics, 1992, ISBN: 9780883188590. [Online]. Available: https://books.google.it/books? id=OsOWJ8iHpmMC

[2] F. Chen, Introduction to Phys. Plasmas and Controlled Fusion, ser. Introduction to Phys. Plasmas and Controlled Fusion v. 1. Springer, 1984, ISBN: 9780306413322.

[3] W. Chen, L. xin Guo, and J. ting Li, "Research on the FDTD method of scattering effects of obliquely incident electromagnetic waves in time-varying plasma sheath on collision and plasma frequencies," Phys. Plasmas, vol. 24, no. 4, p. $042102,2017$.

[4] M. P. Bachynski, "Electromagnetic wave penetration of reentry plasma sheaths," RADIO SCIENCE, vol. 69D, no. 2, 1965.

[5] M. Brambilla, Kinetic Theory of Plasma Waves: Homogeneous Plasmas, ser. International series of monographs on physics. Clarendon Press, 1998, ISBN: 9780198559566.
[6] D. B. Graves, "Plasma processing," IEEE T PLASMA SCI, vol. 22, no. 1, pp. 31-42, 1994.

[7] M. A. Heald and C. B. Wharton, Plasma diagnostic with microwaves. Robert E. Krieger, 1996.

[8] M. Bachynsici and B. Gibbs, "Antenna pattern distortion by an isotropic plasma slab," IEEE Trans. Antennas Propag, vol. 16, p. 583, 1968.

[9] G. Torrisi, D. Mascali, L. Neri, O. Leonardi, G. Sorbello, L. Celona, G. Castro, R. Agnello, A. Caruso, S. Passarello, A. Longhitano, T. Isernia, and S. Gammino, "Microwave frequency sweep interferometer for plasma density measurements in ecr ion sources: Design and preliminary results," Rev. Sci. Instrum., vol. 87, no. 2, 02B909, 2016.

[10] G. Torrisi, G. Sorbello, O. Leonardi, D. Mascali, L. Celona, and S. Gammino, "A new launching scheme for ecr plasma based on twowaveguides-array," Microw. Opt. Technol. Lett., vol. 58, no. 11, pp. 2629-2634, 2016, ISSN: 1098-2760.

[11] R. Geller, Electron Cyclotron Resonance Ion Sources and ECR Plasmas. Taylor \& Francis, 1996, ISBN: 9780750301077.

[12] L. Celona, S. Gammino, G. Ciavola, F. Maimone, and D. Mascali, "Microwave to plasma coupling in electron cyclotron resonance and microwave ion sources (invited)," Rev. Sci. Instrum., vol. 81, no. 2, 02A333, 2010.

[13] G. Torrisi, D. Mascali, G. Sorbello, L. Neri, L. Celona, G. Castro, $\mathrm{T}$. Isernia, and S. Gammino, "Full-wave fem simulations of electromagnetic waves in strongly magnetized non-homogeneous plasma," $J$ ELECTROMAGNET. WAVE., vol. 28, no. 9, pp. 1085-1099, 2014. DOI: $10.1080 / 09205071.2014 .905245$

[14] G Castro, D Mascali, S Gammino, G Torrisi, F. P. Romano, L Celona, C Altana, C Caliri, N Gambino, D Lanaia, R Miracoli, L Neri, and G Sorbello, "Overdense plasma generation in a compact ion source," Plasma. Sourc. Sci. Tech., vol. 26, no. 5, p. 055 019, 2017.

[15] J. J. Simpson and A. Taflove, "A review of progress in FDTD maxwell's equations modeling of impulsive subionospheric propagation below $300 \mathrm{khz}$," IEEE Trans. Antennas. Propag., vol. 55, no. 6 , pp. 1582-1590, 2007, ISSN: 0018-926X.

[16] J. Jia, C. Yuan, R. Gao, Y. Wang, Y. Liu, J. Gao, Z. Zhou, X. Sun, J. Wu, H. Li, and S. Pu, "Propagation of electromagnetic waves in a weakly ionized dusty plasma," J. Phys. D, vol. 48, no. 46, p. 465201 , 2015.

[17] G. Cerri, F. Moglie, R. Montesi, P. Russo, and E. Vecchioni, "FDTD solution of the maxwell-boltzmann system for electromagnetic wave propagation in a plasma," IEEE Trans. Antennas. Propag., vol. 56, no. 8, pp. 2584-2588, 2008, ISSN: 0018-926X.

[18] D. Mascali, G. Torrisi, L. Neri, G. Sorbello, G. Castro, L. Celona, and S. Gammino, "3d-full wave and kinetics numerical modelling of electron cyclotron resonance ion sources plasma: Steps towards selfconsistency," Eur. Phys. J. D, 69, 1, vol. 69, no. 1, p. 27, 2015.

[19] Y. Yu and J. J. Simpson, "An E-J collocated 3-D FDTD model of electromagnetic wave propagation in magnetized cold plasma," IEEE Trans. Antennas. Propag., vol. 58, no. 2, pp. 469-478, 2010, ISSN: 0018-926X.

[20] J. H. Lee and D. K. Kalluri, "Three-dimensional FDTD simulation of electromagnetic wave transformation in a dynamic inhomogeneous magnetized plasma," IEEE Trans. Antennas. Propag., vol. 47, no. 7, pp. 1146-1151, 1999.

[21] R. J. Luebbers, F. Hunsberger, and K. S. Kunz, "A frequencydependent finite-difference time-domain formulation for transient propagation in plasma," IEEE Trans. Antennas. Propag., vol. 39, no. 1, pp. 29-34, 1991.

[22] J. L. Young, "A full finite difference time domain implementation for radio wave propagation in a plasma," Radio Science, vol. 29, no. 6 , pp. 1513-1522, 1994, ISSN: 1944-799X.

[23] T. Ropponen, O. Tarvainen, P. Suominen, T. Koponen, T. Kalvas, and H. Koivisto, "Hybrid simulation of electron cyclotron resonance heating," Nucl. Instr. Meth. Phys. Res. A, vol. 587, no. 1, pp. $115-124$, 2008.

[24] V Mironov, S Bogomolov, A Bondarchenko, A Efremov, and V Loginov, "Some aspects of electron dynamics in electron cyclotron resonance ion sources," Plasma. Sourc. Sci. Tech., vol. 26, no. 7, p. $075002,2017$.

[25] J. Jacquot, V. Bobkov, L. Colas, S. Heuraux, A. Kriivsk, L. Lu, J.M. Noterdaeme, T. S. Team, and A. U. Team, "Full wave propagation modelling in view to integrated icrh wave coupling/rf sheaths modelling," AIP Conference Proceedings, vol. 1689, no. 1, p. 050008 , 2015 . 
[26] M. Brambilla and T. Krcken, "Numer ical sim ula tion of ion cyclotron heating of hot tokamak plasmas; - Nuclear Fusion, vol. 28, no. I 0 , p. 1813,1988

[271 N. Benelli, O. Maj, E. Poli, R. Harvey, J. C. Wright. P. T. Bonoli, C. K. Phillips. A. P. Smimov, E. Valeo. a,1d J. R. Wilso,n "Paraxia 1 wentzel-kramers - brillouin method applfod 10 the lower hybrid wave propagation," Phys. Plasmas, vol. 19, no. 8, p. 0825 10, 2012.

[28] S. Gammino, L. Celona, D. Mascali, G. Castro. G. Torrisi, L. Neri, M. Mazzaglia, G. Sorbello, 0. Leonardi, L. Allegra, G. Calabrese, F. Chine s, G. Gallo, and S. Passe rello. "The flexible plasma trap (fpt) for the production of overdense plasmas," J INSTRUM., vol. 12, no. 07 P07027, 2017.

[291 COMSO L, "Rf module t1ser 's gu id e, version 5.3," (Online!. Available: www.coms b.com

1301 I. Dey a nd S. Bhauachar jee. " Penetratio $n$ and screening of perpendict1larly launched electromagnetic waves through bounded supercritica 1 plasma con fined in mullicusp magnetic field," Phys. Plasmas. vol. I8, no. 2, p. 022101, 2011. 001: I0.1063/1.355 1696

[31] C. A. Lee, G. Chen. A. Y. Are fiev, R. D. Bengtson, and B. N. Breizman * Me.is urements and modeling of radio frequency field structures in a helicon plasma."'Phys. Plasmas, vol, 18, no. 1, p. 013 501, 2011.

[32] M. I. Panevsky. "'Characterizat ion of the resonant electromagnetic mode in helicon discharges," PhD thesis, The University of Texas at Austin. 2003.

[331 E. Yats uka, K. Kinjo, J. Morikawa, and Y. Ogawa, "Radio-frequency electro magne tic field measurements for direcl deleclion of e leclron bemstein waves in a torus plasm a." Re,1*Sci. hzstru m. vol. 80, no. 2. p. 023505,2009

[341 K. Takahashi , T. Kanek o, and R. Halakeyama, "Polarirnl io n-reversalind uced damp ing of lefl- hand pola rized wave on electron cyclotron resonance;' Phys. Rek Lew ., vol. 94, p. $215001,212005$.

1351 Y. Y. Pod(iba, H. P. Laqua, G. B. Warr, M. Schuben, M. One. S. Marse, n F. Wagner, and E. Holzhaue r, " Direct observation of elec1ronberns lein wave heating by o-x-b-mode conversion at low magnetic field in the wega stellarator," Phys. Rev. Leu.. vol. 98, p. 255003. 252007.

[361 M.A. Lieberman and A. J. Lichtenberg.' 'Theory of electron cyclotron resonance heating. ii. long time and slochas 1 ic effects," Phys. Plasmas, vol. 15 , no. 2 , p. 125 , 1973 .

[37] R Racz D Mascali, S Biri, C Caliri G Castro, A Galala' S Gammino, L Neri, J Palinkas, F. P. Roman,o and G Ton'isi, "Electron cyclotron resonance ion source plasma characterization by e nergy $\mathrm{d}$ isper s ive $\mathrm{x}$ ray imag ing," Plasma. S0111Y: Sci. Tech., vol. 26, no. 7. p. 075011. 2017.

[38] B. P. Cluggish and J.-S. Kim, "Modeling of wave propagation and

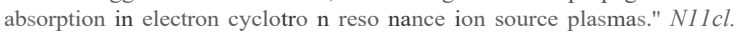
Inst,: Meth. Phys. Res. il, vol. 664. no. I, pp. 84 - 97, 20 12, ISSN: 01689002

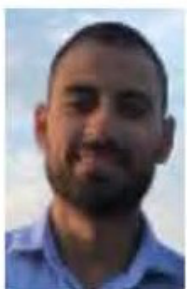

Giuseppe Tor risi Giuseppe Torrisi was born in Cata nia, Ital y. in I987. He received lhe M.S. Laurea degree (summa cum laude) in telecommunication e ngineer ing from the University of Catania (Catania Italy) in $20 \mathrm{II}$ and the $\mathrm{Ph} . \mathrm{D}$ degree in infornlalion e ngineer ing from Universila Mediterranea of Reggio Calab ria (Regg io Calabria. Italy) in 2016. $\mathrm{He}$ is curren tly a temporary staff researcher of the lstilu10 Nazionale di Fisica Nucleare (! NFN) at Laboraiori Nazionali del S ud (LNS), Catania. His scientific aclivily is concerned with electromagnetic propagation in microwave generated plasmas produced by ion so urces for part icle accelerawrs. In particular, he is prese ntly focused on the development or innovative microwave schemes for plas ma heating and diagnoslics. $\mathrm{He}$ is author of more than 50 papers published on peer reviewed scientific journa ls or inte rnat ional conference proceeding. Dr. Torrisi was the recipien1 of the Best paper prize during the XXI Riunione Nazionale di Elettromagnetismo in 2016. the France-5coResmini award for the best PhD Thes is on accelera tors and new tec hnologie s fro $\mathrm{m}$ the INFN Co mm. V in 2017, the Yo ung Sc ientist Award at the 2nd URSI Atlantic Radio Science Meeting and the Sannino prizeawarded by the Italian Electrom agnet ics Society for the best work on $\mathrm{mm}$-waves and microwave circuits in 2018 .

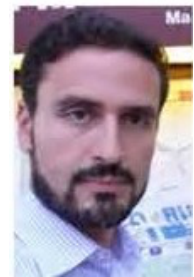

David Mascali David Mascali was born in Catania Italy, in July 1981. He received the Maste $\mathrm{r}$ degree (in 2005) and the $\mathrm{PhD}$ (in 2009) cum laude in Experime ntal Physics from the Universil degli Studi di Catania. Italy. He is cu, ently a StafT Researcher at the Laboralori Naz ionali <lei S ud. Is t itu to Nazionale di Fis ica Nucleare (CNFN-LNS). leading the Ion Beam s Production team inside 1he Accelerators Division. $\mathrm{He}$ is the spokesperson of the PANDORA ex perime nt, s upponed by INPN, aiming at interdisciplinary research in AsLroph ysic s and Nuclear Physics in laboratory plasmas, and of the Grant "IRIS" for inn ova tive resonators for ECR ion source s. His research activity is mainly focused on plasma physics, plasma-based ion sources science and technology, plasma diagnostics. laser produced plasmas. In 2009 he received, in Firenze. the National F. Resmini Prize for innovative research in Accelerator Phys ics. In 20 ,I2 he was awarde d in Sidney, Australia, by 1.he international "R. Geller" prize for oumanding researches in Ton Source s Science and Technology. He is a member of he Scientific Programme Committee of Int. Conr. o n Ion So $11 \mathrm{rc}$ es since 2013. Since 2017 he teaches classes of "Plasma Phy s ics for [n1erdi scl iplinary Research" al the PhD cot1rse of the Cata 11 ai University. He gave seve ral invited talks and seminars in Europe. USA and Japan, authoring more than 80 peerreviewed papers and more than 70 conference proceedings.

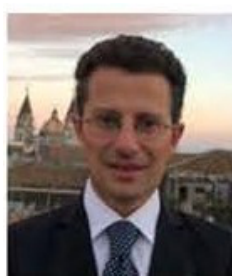

Gino Sorbello Gino Sorbello received the degree in Electro nics Engineeri ng cum laude at Lh e Un ivers ity of Cata nia, Italy. in I996, and the Ph.D. degree in Electronics and Communications Enginee ring al th e Po lytec hni c ln slitule of M ilan in 2000 . In 2002 he became Assistant Professor of Electromagnetic Fields al University of Catania. Since 2014. he is Associate Professor of Electromagnetic fields at the Dipanimento di lngegne ria Ele1trica. EletLronica e lnformatic, a University of Catania. His current resea rch interes ts include the study of single- mode solid-state waveguide lasers and amplifiers, integrated optics, the developmen of planar antennas and ultra-wideband co mpact antennas and antenna arrays, the study of microwave devices and compurational electromagnetism with a special interest in RF-plasma inte ractio ns and panicle accelerators. Gino Sorbello autho red more than 50 scientific papers and more than 30 conference proceedings.

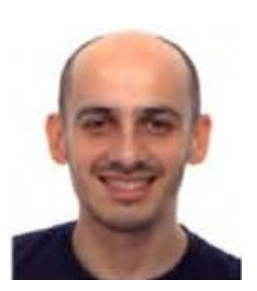

Giuseppe Castro Giuseppe CasLro rece ived the ma ster degree in physics at the University of Catania, Italy. in 2008, and tile Ph.D. degree in physics in 2013 in the same university with a thesis on the Study of innovative plasma heating methods and applications 10 high current ion sources. From 2015 he is adjunct professorof Ins1ilu1esof physics I al the depan ment of physics of the University of Catania. $\mathrm{He}$ is involved from the beginning of his career in the study of plasma physics devoted 10 application in the field of nuclear physics and ion sources and in the development of innovative methods of plasma heating and plas ma diagno stics. Among the, $\mathrm{m}$ it is wonh 10 cite the development of the optical emission spectroscop y, Langmuir probe. X-ray diagnostics and interfero-polarime1ryfor the magnetized $\mathrm{J}>$ lasma of io $\mathrm{n}$ source, $\mathrm{s}$ the studie $\mathrm{s}$ on the elec tro $\mathrm{n}$ Bernstein waves heatiJ1g and the deve lopme nt. a long the yea rs. of different proton and ion source for acce lerator, as VIS. PS-ESS and AISHa. In these fields. he authored more than 100 between peer-reviewed and proceeding $\mathrm{s}$ and gave several talks in the most important topic conferences. 


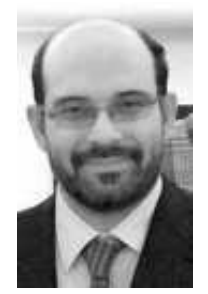

Luigi Celona L.G. Celona received the degree in Electronic Engineering at the University of Catania, in 1995 and he joined the Istituto Nazionale di Fisica Nucleare in 1996, at the Laboratori Nazionali del Sud (INFN-LNS), becoming Technological Engineer (Tecnologo) in 1998 and Principal Technological Engineer (Primo Tecnologo) in 2006. His main field of activity covers all the aspects of the production of singly and highly charged beams together with their acceleration to increase the performances of Particles Accelerators for Nuclear and Applied Physics. Experienced in all the design stages of an ion source: from mechanical design and manufacturing through the installation and final commissioning, he is also active in research and development, proposing different innovative concepts concerning the role of microwaves in the development of ECR and microwave ion sources. He is a member of the INFN Machine Advisory Committee as ion source expert and of the Conseil Scientifique et Technique du Dpartement des Acclrateurs, de Cryognie et de Magntisme of CEA. He was member of the steering committee of the SPES project and, in the European framework, he was a referee committee member of ARES and EMILIE projects to coordinate the R\&D activities on ECR ion sources of the major European physics labs. He was the technical and scientific responsible of a joint-venture between INFN-LNS and some SMEs to design, realize and test a new hybrid ion source for Hadrontherapy named AISHa: two ion sources have been manufactured and successfully commissioned. He is the Leader of the design, manufacturing and commissioning of the high intensity proton sources along with the low energy beam transfer line for the European Spallation Source (ESS). The outstanding commissioning results fully comply the requirements given. The first source has been successfully installed in its final position at ESS site at the beginning of 2018 as a first part of ESS linac. He has also designed and built other types of ion and plasma sources, as the microwave discharge ion sources named MIDAS, TRIPS and VIS, for high efficiency ionization of the reaction products originating from an ISOL target and for intense monocharged production of light ions. During the period 2004-2007 he focused his efforts on the EXCYT radioactive beam facility, coordinating the installation, the commissioning and permitting to deliver the $8 \mathrm{Li}$ beam for the first experiments. He also worked on the development of the K-800 Superconducting Cyclotron bunching system contributing to the optimization of the cyclotron in the years 1995-1998 and to the axial injection beamline. He was the chair of the last International Workshop on ECR ion sources authoring more than 100 peer-review journals and more than 150 conference proceedings.

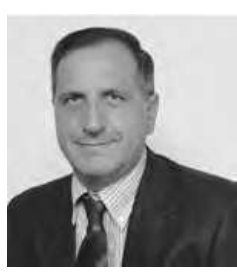

Santo Gammino Santo Gammino was born in Riposto, Italy, in 1963. He received the "Laurea" degree in Physics from the University of Catania, Italy in 1987 and he joined the Istituto Nazionale di Fisica Nucleare in 1988, at the Laboratori Nazionali del Sud in Catania (INFN-LNS), becoming Research Physicist in 1990, Principal Research Physicist in 2002 and Director of Research in 2009. His research has been focused to the production of monocharged and highly charged beams, and their acceleration. He proposed different innovative concepts for the development of ECR (electron cyclotron resonance) ion sources, and he actively worked on the construction of many different ECR ion sources. In particular his project SERSE came in operation in 1997 and for many years it was on the forefront for the production of highly charged ions. This activity has taken in the last years to the development of the so-called "3rd generation ECRIS and of different ion source (e.g. AISHA). He has also designed and built other types of ion sources, as laser ion sources and the microwave discharge ion sources named MIDAS, TRIPS and VIS, for high efficiency ionization of recoils and for intense monocharged beam production respectively. He also worked during the Nineties on the development of the K-800 Superconducting Cyclotron and on the design and construction of the EXCYT radioactive beam facility at INFN-LNS. Since 2010 he has been leader of the WP3-Normal conducting Linac for the European Spallation Source and for this facility he designed the high intensity proton source PSESS. He is also responsible for INFN in-kind contribution, including the Drift Tube Linac and the Medium Beta Superconducting Cavities, both under construction. Dr. Gammino served as a member of the National Committee of INFN for the Technological Research from 1996 to 2002 and for a third term from 2008 to 2012. In 2012 he has been charged as responsible for the R\&D of Accelerators at INFN-LNS. He has been involved in the preparation of the NUPECC Long Range Plan 2010 and has been member of different Committees in Italy and abroad. Dr. Gammino has been author of more than two hundreds peer reviewed papers and more than three hundreds contributions to conferences and other publications. 